\title{
Development of novel rat model for high-fat and high-cholesterol diet-induced steatohepatitis and severe fibrosis progression in SHRSP5/Dmcr
}

\author{
Kazuya Kitamori · Hisao Naito $\cdot$ Hazuki Tamada $\cdot$ Miya Kobayashi - Daisuke Miyazawa \\ Yuko Yasui · Kunihiro Sonoda $\cdot$ Satoru Tsuchikura $\cdot$ Naomi Yasui $\cdot$ Katsumi Ikeda \\ Takashi Moriya $\cdot$ Yukio Yamori $\cdot$ Tamie Nakajima
}

Received: 30 March 2011 / Accepted: 31 July 2011/Published online: 19 August 2011

(c) The Japanese Society for Hygiene 2011

\begin{abstract}
Objectives Patients with nonalcoholic fatty liver disease are increasing worldwide, and preventive measures are an urgent need and primary concern today.

Aim This study aimed to develop and clarify the usefulness of the SHRSP5/Dmcr rat, derived from a stroke-prone spontaneously hypertensive rat, as a novel animal model for time-course analysis of steatohepatitis and the severe fibrosis progression often observed in the disease.

Methods Ten-week-old male SHRSP5/Dmcr rats were divided into six groups: half were fed a high-fat and highcholesterol-containing diet (HFC diet), and the others the control, stroke-prone (SP) diet for 2, 8, and 14 weeks.

Results The HFC diet significantly increased serum transaminase and gamma glutamyl transpeptidase
\end{abstract}

K. Kitamori $\cdot$ H. Naito $\cdot$ H. Tamada

T. Moriya $\cdot$ T. Nakajima $(\bowtie)$

Department of Occupational and Environmental Health,

Nagoya University Graduate School of Medicine,

65 Tsurumai-cho, Showa-ku, Nagoya 466-8550, Japan

e-mail: tnasu23@med.nagoya-u.ac.jp

K. Kitamori - H. Tamada - M. Kobayashi - D. Miyazawa

Y. Yasui · K. Sonoda

College of Human Life and Environment,

Kinjo Gakuin University, Nagoya, Japan

S. Tsuchikura

Disease Model Cooperative Research Association,

Kyoto, Japan

N. Yasui · K. Ikeda

School of Pharmacy and Pharmaceutical Sciences,

Mukogawa Women's University, Nishinomiya, Japan

Y. Yamori

Institute for World Health Development,

Mukogawa Women's University, Nishinomiya, Japan activities, tumor necrosis factor alpha levels, and serum and hepatic total cholesterol levels over time. In contrast, this diet decreased serum albumin, glucose, and adiponectin levels throughout or the later stage of the feeding period, but did not influence serum insulin levels. Histopathologically, the HFC diet increased microvesicular steatosis, and focal or spotty necrosis with lymphocyte infiltrations were observed in the liver at 2 weeks, macrovesicular steatosis, ballooned hepatocytes with MalloryDenk body formation in some, and multilobular necrosis and fibrosis at 8 weeks. Interestingly, this fibrosis formed a honeycomb network at 14 weeks. These changes are very similar to those observed in patients with non-alcoholic steatohepatitis.

Conclusions SHRSP5/Dmcr rats appear to be a useful model for analyzing the time-dependent changes of HFC diet-induced steatohepatitis and fibrosis progression.

Keywords SHRSP5/Dmcr · High-fat and highcholesterol-containing diet $\cdot$ Non-alcoholic fatty liver disease $\cdot$ Non-alcoholic steatohepatitis $\cdot$ Fibrosis

$\begin{array}{ll}\text { Abbreviations } \\ \text { ALT } & \text { Alanine transaminase } \\ \text { AST } & \text { Aspartate transaminase } \\ \text { CCl4 } & \text { Carbon tetrachloride } \\ \text { DMN } & \text { Dimethylnitrosamine } \\ \text { FFA } & \text { Free fatty acid } \\ \gamma \text {-GTP } & \gamma \text {-Glutamyl transpeptidase } \\ \text { H\&E } & \text { Hematoxylin and eosin } \\ \text { HOMA-IR } & \text { Homeostasis model assessment-insulin } \\ & \text { resistance } \\ \text { HFC } & \text { High fat and cholesterol-containing } \\ \text { NAFL } & \text { Non-alcoholic fatty liver } \\ \text { NAFLD } & \text { Non-alcoholic fatty liver disease }\end{array}$




$\begin{array}{ll}\text { NAS } & \text { Non-alcoholic fatty disease activity score } \\ \text { NASH } & \text { Non-alcoholic steatohepatitis } \\ \text { SP } & \text { Stroke-prone } \\ \text { SHRSP } & \text { Stroke-prone spontaneously hypertensive rat } \\ \text { TC } & \text { Total cholesterol } \\ \text { TG } & \text { Triglyceride } \\ \text { TNF } \alpha & \text { Tumor necrosis factor } \alpha \\ \text { TP } & \text { Total protein }\end{array}$

\section{Introduction}

Non-alcoholic fatty liver disease (NAFLD) includes a progressive liver disorder from non-alcoholic fatty liver (NAFL) to non-alcoholic steatohepatitis (NASH) without a history of alcohol abuse, which is increasingly observed as a comorbidity of the metabolic syndrome phenotype [1]. During the progression of NAFLD, hepatocyte steatosis and necrosis, infiltration of inflammatory cells, and fibrosis consistently appear, and the fibrosis results in hepatic cirrhosis or, finally, in tumor formation. Indeed, of 73 patients with NASH, 25\% developed cirrhosis [2]; of 82 of those with NASH, 7\% hepatocellular carcinoma [3]. Actually, 9\% of 382 patients with NASH were also diagnosed with hepatocellular carcinoma [4]. On the other hand, the percentage of NASH in patients with NAFLD was approximately $60 \%$ [5]. Thirty-six percent of the patients with NAFLD were also found to have severe fibrosis by biopsy [6].

To prevent an increase in the number of patients with NAFLD, it is important to clarify the mechanism that leads to NAFLD. Many approaches have been conducted; a highfat and high-cholesterol (HFC) diet has been used as an essential causative factor to induce NAFLD in rodents [7]. In this animal model, the diet induced lipid accumulations and an increase of pro-inflammatory cytokines such as tumor necrosis factor $\alpha(\mathrm{TNF} \alpha)$ in the livers of the mice. In addition, the feeding of a methionine- and choline-deficient diet was shown to induce the development of fibrosis $[8,9]$. However, obvious fibrosis with bridging that forms a honeycomb network, which has been occasionally noted in patients with NASH [10, 11], could not be observed. On the other hand, analysis of the mechanism of hepatic fibrosis has been conducted using dimethylnitrosamine (DMN) or carbon tetrachloride $\left(\mathrm{CCl}_{4}\right)$-administered animal models [12-14]. In these chemically induced models, a fibrotic response was manifested, but those models could not help to identify the mechanism in the development from NAFL to NASH due to the lack of a progressive pathology. In other words, no dietary-induced models have been developed for observing time-dependent progression of NASH with bridging fibrosis.
The stroke-prone spontaneously hypertensive rat, SHRSP5/Dmcr, formerly called the arteriolipidosis-prone rat, is the 5th substrain of SHRSP registered at the National Bio Resource Center. In order to develop a model of quickly accumulating arterial fat deposition, that is, arteriosclerosis, in response to an HFC diet, the new substrain has been produced by selective brother-sister inbreeding of SHRSP with stronger hypercholesterolomic responses (about $600-900 \mathrm{mg} / \mathrm{dl}$ for females and $300-600 \mathrm{mg} / \mathrm{dl}$ for males) to an HFC diet and water ad libitum for 1 week [15]. Acute arterial fat deposits were microscopically observed in mesenteric arteries [16]. At the 36th generation, pregnant female rats were given from Y. Yamori, Emeritus Professor of Kyoto University, and the offspring were obtained by caesarean delivery, that is, specific pathogen-free. They were maintained up to the 46th generation at Kinjo Gakuin University. Although the original aim was to develop the arteriosclerosis model rat in response to an HFC diet, we had found marked enlargement and whitish color of the liver of the 47th generation. Therefore, we focused our attention on the analysis of liver damage caused by an HFC diet. SHRSP/5Dmcr strain was characterized to have lower hypertension and stroke incidences than those of the parent SHRSP one during the inbreeding.

In order to clarify whether the SHRSP5/Dmcr might be a pathologically appropriate novel rat model showing the progression from steatohepatitis to severe fibrosis, we first investigated the histopathological and biochemical changes of liver function, lipid metabolism, inflammation, and fibrosis over time in the rats fed an HFC diet for 2, 8, and 14 weeks, and compared the findings with those in SHRSP5/Dmcr fed a control, stroke-prone (SP), diet (Funabashi Farm, Chiba, Japan).

\section{Materials and methods}

Animals and feeding of HFC diet

This study was conducted according to the Guidelines for Animal Experiments of the Kinjo Gakuin University Animal Center. All SHRSP5/Dmcr (49th generation) rats were housed in a temperature- and light-controlled environment $\left(23 \pm 2{ }^{\circ} \mathrm{C}, 55 \pm 5 \%\right.$ humidity, 12 -h light/dark cycle), and maintained on a control chow (SP diet) [17], the composition of which is shown in Table 1, and tap water ad libitum. After 1 week on the HFC diet (Funabashi Farm) [15], males and females with high cholesterol levels (about $300-700 \mathrm{mg} / \mathrm{dl}$ for females and $100-300 \mathrm{mg} / \mathrm{dl}$ for males, respectively) were mated, and we thus obtained 36 male offsprings that at the age of 10 weeks were assigned to the following six groups: three groups were fed the SP diet for 
Table 1 Nutrient components of SP [16] and HFC-containing diets [15] (weight \%)

\begin{tabular}{lcc}
\hline & Dietary groups \\
\cline { 2 - 3 } & SP diet & $\begin{array}{l}\text { HFC-containing } \\
\text { diet }\end{array}$ \\
\hline Feed formulation rate & 100 & 68 \\
SP diet & - & 25 \\
Palm oil & - & 5 \\
Cholesterol & - & 2 \\
Cholic acid & & \\
Ingredients & 20.8 & 14.1 \\
Crude protein & 4.8 & 35.3 \\
Crude lipid & 3.2 & 2.2 \\
Crude fiber & 5.0 & 3.4 \\
Crude ash & 8.0 & 5.4 \\
Moisture & 58.2 & 39.6 \\
Carbohydrate & & \\
\hline
\end{tabular}

Total energy was $4.14 \mathrm{kcal} / \mathrm{g}$ in SP diet and $5.72 \mathrm{kcal} / \mathrm{g}$ in HFCcontaining diet

2,8 , and 14 weeks (control group) and the remaining were fed the HFC diet for 2, 8, and 14 weeks. Calorie intakes were not changed in the SP diet group throughout the experiment $(142 \pm 11,139 \pm 2$ and $140 \pm 4 \mathrm{kcal} /$ day $/ 2$ rats at 2,8 , and 14 weeks, respectively, $n=3$ cages), while the intakes time-dependently decreased in the HFC diet group $(152 \pm 22,97 \pm 26$ and $55 \pm 12 \mathrm{kcal} / \mathrm{day} / 2$ rats at 2,8 , and 14 weeks, respectively, $n=3$ cages). Since the calorie intake in the HFC diet group was smaller than that of the SP diet group at the first week, this may reflect on the lower body weight in the former group rather than the latter group at 2 weeks. Accordingly, sodium intakes in HFC diet groups were lower than that of SP diet groups $(134 \pm 11$, $131 \pm 1.7$ and $128 \pm 4.0 \mathrm{mg} /$ day/2 rats in SP diet for 2,8 , and 14 weeks, respectively; $111 \pm 16,71 \pm 19$, and $41 \pm 8.8 \mathrm{mg} / \mathrm{day} / 2$ rats in the HFC diet for 2,8 , and 14 weeks, respectively, $n=3$ cages). After $18-20 \mathrm{~h}$ fasting from the last feeding, all rats were killed under anesthesia by pentobarbital $(70 \mathrm{mg} / \mathrm{kg}$ ), and the blood and livers were removed. A part of each liver was fixed in $4 \%$ buffered paraformaldehyde, and the remaining was stored at $-80^{\circ} \mathrm{C}$. Serum was collected after centrifuging the blood at $3,500 \times g$ for $10 \mathrm{~min}$ and stored at $-80^{\circ} \mathrm{C}$ until use.

Body weight, blood pressure, and biochemical assay

Before the rats were killed, we measured their systolic blood pressure by the tail-cuff method and then checked their body weight. Serum glucose, total protein (TP), albumin, total cholesterol (TC), triglyceride (TG), aspartate transaminase (AST), alanine transaminase (ALT), free fatty acid (FFA), type IV collagen $7 \mathrm{~S}, \gamma$-glutamyl transpeptidase (GTP) and insulin levels were measured by SRL, Inc. (Tokyo, Japan). Serum TNF $\alpha$ and adiponectin were measured using kits from R\&D Systems (Minneapolis, MN, USA) and Otsuka pharmaceuticals (Tokyo, Japan), respectively.

Lipid from livers was extracted using the method of Folch et al. [18]. TG and TC in the liver were measured using kits of TG-IE and T-Cho IE (Wako, Osaka, Japan), respectively.

\section{Histopathological analysis}

Small blocks of liver tissues from each rat fixed in $4 \%$ buffered paraformaldehyde were embedded in paraffin and sliced into $4-\mu \mathrm{m}$ sections. Tissue sections were stained with hematoxylin and eosin (H\&E) or Azan, and examined under a light microscope using the DMD108 (Leica, Wetzlar, Germany). Steatosis, inflammation, hepatocyte ballooning [19], macrovesicular steatosis [20], and fibrosis [21] were numbered according to the following classifications: (1) grade of steatosis: 0 , none; 1 , mild (5-33\% of parenchymal involvement by steatosis); 2 , moderate (33-66\%); 3, severe (>66\%); (2) lobular inflammations: 0 , none; 1 , mild; 2 , moderate; 3 , severe; (3) hepatocyte ballooning: 0 , none; 1 , few ballooned cells; 2, many cell-prominent ballooning; (4) macrovesicular steatosis: same as steatosis scoring; and (5) fibrosis stage: 1 , zone 3 perisinusoidal/pericellular fibrosis; 2 , zone 3 perisinusoidal/pericellular fibrosis with focal or extensive periportal fibrosis; 3, zone 3 perisinusoidal/pericellular fibrosis and portal fibrosis with focal or extensive bridging fibrosis; 4 , cirrhosis. Then, the NAFLD activity scores (NAS) were calculated according to the NASH Clinical Research Network scoring system [19], which has been applied to highfat feeding rats [20].

\section{Statistical analysis}

Comparisons were made using two-way analysis of variance for the diet fed and duration of the feeding, followed by the Tukey-Kramer HSD post hoc test of the differences in duration of the feeding of SP or HFC diet, respectively, and the $t$ test between groups fed SP and HFC diets at each point. Values of $p<0.05$ were considered to indicate statistical significance.

\section{Results}

Changes in body and liver weight, and blood pressure

Body weight increased in the group fed the SP diet with the increasing duration of the feeding, while the increase was 
moderate in the group fed the HFC diet compared to that of the SP group (Table 2). Liver weight did not change in the SP diet group throughout the duration of the diet feeding, while in the HFC diet group, the weight significantly increased at 2-, 8-, and 14-week feedings compared to that of the SP diet. There were no changes in blood pressure in the SP diet group during the experimental period, while the HFC diet group exhibited significantly decreased blood pressure at 14 weeks compared to the SP diet.

\section{Serum biochemical assay}

TP levels were not changed by HFC-diet feeding in the follow-up periods, though the levels in the SP-diet group were lower at 2 weeks than those at 8 and 14 weeks. The HFC diet significantly decreased albumin levels at 8 and 14 weeks, which were also lower than those in the respective SP-diet group. HFC diet did not influence insulin levels in the follow-up periods. Glucose levels were always lower in the HFC-diet group than in the SP-diet group in the follow-up periods. The HFC diet did not influence FFA levels except at 8 weeks. Interestingly, there were no differences in TG levels between the SP and HFC diet in the follow-up periods, though the levels were significantly higher in HFC-diet group at 14 weeks than those at 8 weeks. HFC diet dramatically increased serum TC levels compared to those of the SP diet at all observed periods, and the levels markedly increased by more than 10 -fold at 14 weeks compared to the levels at 2 weeks.

HFC diet increased AST and ALT activities at 2 weeks compared to those in the SP diet, and further increased at 8 (AST, 5.2-fold and ALT, 4.2-fold) and 14 weeks (9.5- and 7.7-fold, respectively) in a duration-dependent manner. Serum $\gamma$-GTP levels were under the detection limit (3 IU/l) in all rats fed the SP diet in all follow-up periods and those fed the HFC diet for 2 weeks. However, the values were over 3 IU/1 in all rats fed the HFC diet for 8 and 14 weeks. Moreover, the levels in the HFC diet group at 14 weeks were higher than in the group at 8 weeks. There were no changes in homeostasis model assessment-insulin resistance (HOMA-IR) (data not shown) calculated by the insulin and glucose values of all rats.

The SP diet did not influence TNF $\alpha$ levels, however, the HFC diet significantly increased these levels at 2 weeks, and further increased at 14 weeks of HFC. The HFC diet increased serum adiponectin levels at 2 weeks, whereas the levels decreased at 14 weeks compared to the levels of the SP diet group.

Table 2 Body and liver weights, blood pressure, and various serum levels in rats fed SP- and HFC-containing diets

\begin{tabular}{|c|c|c|c|c|c|c|}
\hline & \multicolumn{3}{|l|}{ SP diet } & \multicolumn{3}{|l|}{ HFC diet } \\
\hline & 2 weeks & 8 weeks & 14 weeks & 2 weeks & 8 weeks & 14 weeks \\
\hline Body weight (g) & $263 \pm 19$ & $314 \pm 20^{\mathrm{b}}$ & $327 \pm 23^{\mathrm{b}}$ & $243 \pm 13^{\mathrm{a}}$ & $268 \pm 14^{\mathrm{a}}$ & $277 \pm 19^{\mathrm{a}, \mathrm{b}}$ \\
\hline Blood pressure (mmHg) & $173 \pm 13$ & $175 \pm 6.7$ & $180 \pm 12$ & $161 \pm 14$ & $168 \pm 18$ & $136 \pm 13^{\mathrm{a}, \mathrm{b}, \mathrm{c}}$ \\
\hline Liver weight (g) & $7.3 \pm 0.72$ & $8.3 \pm 0.76$ & $8.7 \pm 0.87$ & $11.6 \pm 3.8^{\mathrm{a}}$ & $34.6 \pm 3.8^{\mathrm{a}, \mathrm{b}}$ & $37.5 \pm 4.9^{\mathrm{a}, \mathrm{b}}$ \\
\hline $\mathrm{TP}(\mathrm{g} / \mathrm{dl})$ & $5.8 \pm 0.21$ & $6.3 \pm 0.17^{b}$ & $6.2 \pm 0.25^{\mathrm{b}}$ & $6.3 \pm 0.12^{\mathrm{a}}$ & $6.5 \pm 0.24$ & $6.4 \pm 0.25$ \\
\hline Albumin (g/dl) & $4.1 \pm 0.08$ & $4.4 \pm 0.10$ & $4.2 \pm 0.15$ & $4.1 \pm 0.12$ & $3.0 \pm 0.21^{\mathrm{a}, \mathrm{b}}$ & $3.1 \pm 0.12^{\mathrm{a}, \mathrm{b}}$ \\
\hline Glucose (mg/dl) & $132 \pm 9.3$ & $158 \pm 18^{\mathrm{b}}$ & $144 \pm 16$ & $111 \pm 17^{\mathrm{a}}$ & $95 \pm 7.4^{\mathrm{a}}$ & $100 \pm 7.9^{\mathrm{a}}$ \\
\hline Insulin (ng/ml) & $0.52 \pm 0.28$ & $1.27 \pm 0.68$ & $0.62 \pm 0.27$ & $0.75 \pm 0.43$ & $0.78 \pm 0.34$ & $0.40 \pm 0.38$ \\
\hline FFA $(\mu \mathrm{EQ} / \mathrm{l})$ & $452 \pm 64$ & $432 \pm 79$ & $477 \pm 140$ & $566 \pm 174$ & $328 \pm 51^{\mathrm{a}}$ & $502 \pm 384$ \\
\hline TG (mg/dl) & $36 \pm 13$ & $37 \pm 9.3$ & $42 \pm 7.7$ & $41 \pm 5.4$ & $29 \pm 11$ & $79 \pm 63^{\mathrm{c}}$ \\
\hline $\mathrm{TC}(\mathrm{mg} / \mathrm{dl})$ & $51 \pm 1.6$ & $61 \pm 5.2$ & $69 \pm 2.8$ & $129 \pm 52^{\mathrm{a}}$ & $266 \pm 157^{\mathrm{a}}$ & $1593 \pm 884^{\mathrm{a}, \mathrm{b}, \mathrm{c}}$ \\
\hline AST (IU/l) & $122 \pm 12$ & $105 \pm 10$ & $118 \pm 21$ & $144 \pm 11^{\mathrm{a}}$ & $545 \pm 209^{\mathrm{a}, \mathrm{b}}$ & $1121 \pm 320^{\mathrm{a}, \mathrm{b}, \mathrm{c}}$ \\
\hline ALT (IU/l) & $47 \pm 3.5$ & $52 \pm 4.1$ & $50 \pm 5.5$ & $94 \pm 15^{\mathrm{a}}$ & $220 \pm 66^{\mathrm{a}, \mathrm{b}}$ & $387 \pm 87^{\mathrm{a}, \mathrm{b}, \mathrm{c}}$ \\
\hline Type IV collagen $7 \mathrm{~S}(\mathrm{ng} / \mathrm{ml})$ & $3.7 \pm 0.36$ & $3.7 \pm 0.28$ & $3.5 \pm 0.23$ & $3.7 \pm 0.22$ & $3.7 \pm 0.33$ & $4.7 \pm 0.27^{\mathrm{a}, \mathrm{b}, \mathrm{c}}$ \\
\hline$\gamma$-GTP (IU/l) & $1.5 \pm 0^{\mathrm{d}}$ & $1.5 \pm 0^{\mathrm{d}}$ & $1.5 \pm 0^{\mathrm{d}}$ & $1.5 \pm 0^{\mathrm{d}}$ & $10.3 \pm 2.0^{\mathrm{a}, \mathrm{b}}$ & $13.8 \pm 2.9^{\mathrm{a}, \mathrm{b}, \mathrm{c}}$ \\
\hline $\mathrm{TNF}-\alpha(\mathrm{pg} / \mathrm{ml})$ & $1.3 \pm 1.9$ & $0.5 \pm 0.49$ & $2.0 \pm 2.0$ & $7.2 \pm 1.9^{\mathrm{a}}$ & $14.5 \pm 3.1^{\mathrm{a}}$ & $15.8 \pm 6.2^{\mathrm{a}, \mathrm{b}}$ \\
\hline Adiponectin $(\mu \mathrm{g} / \mathrm{ml})$ & $5.4 \pm 0.44$ & $5.2 \pm 1.0$ & $6.3 \pm 0.79$ & $7.4 \pm 0.95^{\mathrm{a}}$ & $4.9 \pm 2.3$ & $4.0 \pm 0.58^{\mathrm{a}, \mathrm{b}}$ \\
\hline
\end{tabular}

Each figure represents the mean $\pm \mathrm{SD}(n=6)$

${ }^{a}$ Significant differences were observed between rats fed SP and HFC diets for the same period $(p<0.05)$

b Significantly different from rats fed the respective diets for 2 weeks $(p<0.05)$

c Significantly different from rats fed the respective diets for 8 weeks $(p<0.05)$

${ }^{\mathrm{d}}$ When under the limit (3.0 IU/l), the values used were half of the limit 
Serum collagen level

As an index of hepatic fibrosis [22], we measured type IV collagen 7S levels of rats fed the SP and HFC diets. There were no differences in the levels between both diet groups at feeding periods of 2 and 8 weeks. In contrast, the values in rats fed the HFC diet significantly increased after feeding for 14 weeks.

Macroscopic and microscopic liver pathology

Macroscopically, the livers of rats fed the HFC diet for 2 weeks became white in color compared to those fed the SP diet (Fig. 1). The whitish color in the HFC group further developed with a longer feeding duration. After feeding for 14 weeks, the surface of the liver appeared to be uneven and hard. In contrast, there was no obvious change in the liver of rats fed the SP diet during all feeding periods.

In the liver of rats fed the SP diet, mild lymphocyte infiltrations into the lobules were observed throughout the feeding periods, but no steatosis and hepatocyte ballooning were observed (Fig. 2a-c, g-i). In the liver of rats fed the HFC diet for 2 weeks, microvesicular steatosis and lymphocyte infiltrations were observed in the whole area, and focal or spotty necrosis with lymphocyte infiltrations were observed in the interpericentral area (Fig. 2d, j), all of which became more severe at 8 weeks. However, the microvesicular steatosis accounted for most of the steatosis (Fig. 2e). Additionally, disruption of the lobular structure, macrovesicular steatosis, ballooned hepatocytes, and multilobular necrosis were observed in the interpericentral area (Fig. 2k). Interestingly, eosinophilic Mallory-Denk bodies were occasionally observed in the ballooned hepatocytes (Fig. 2k). At 14 weeks on the HFC diet, macrovesicular steatosis was observed more predominantly than $\mathbf{a}$

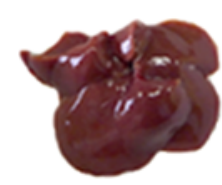

d

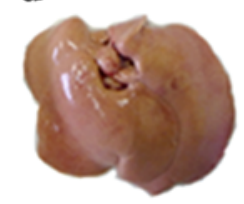

b

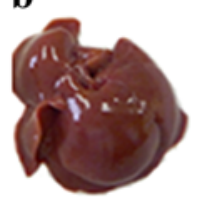

e

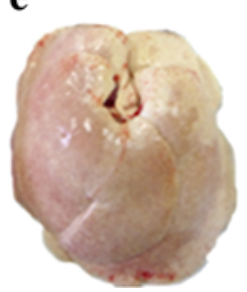

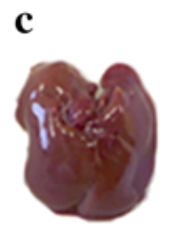

f

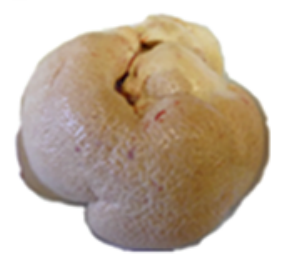

Fig. 1 Macroscopic photos of liver in rats fed SP diet for 2 weeks (a), 8 weeks (b), and 14 weeks (c), or HFC diet for 2 weeks (d), 8 weeks (e), and 14 weeks (f) microvesicular in the livers of rats, and increased in severity over steatosis at 8 weeks (Fig. 2f). Ballooned hepatocytes with eosinophilic Mallory-Denk bodies, multilobular necrosis with collapse were also observed by the wide field-of-view of microscope (Fig. 21).

Pericellular and perivenular fibrotic changes were slightly observed in the liver of rats fed the HFC diet at 2 weeks (Fig. 3d). At 8-week feeding of the HFC diet, bridging fibrosis was observed in the area along with sever macrovesicular steatosis, inflammatory cell infiltrations and ballooned hepatocytes (Fig. 3e). Interestingly, the honeycomb networks were structured by fiber, and different-sized nodules were also found in all microscope fields in the livers of rats fed the HFC diet for 14 weeks (Fig. 3f). Additionally, multilobular necrosis was observed along with honeycomb fibrosis. In contrast, no such changes were observed in the livers of rats fed the SP diet during the follow-up periods (Fig. 3a-c).

\section{NAS evaluation}

We tried to score the hepatocyte changes induced by the HFC diet according to Kleiner et al. [19] and Fisher et al. [23], and calculated the NAS and fibrosis scores (Table 3). The mean steatosis score was 2.9 at 2 weeks in rats fed the HFC diet. This score remained unchanged with further feeding of the diet. Significant macrovesicular steatosis appeared at 8 weeks, and the score accounted for more than half that of steatosis at 14 weeks. Although slight lobular inflammation was observed in the liver of the rats fed the SP diet, it did not increase even after longer feeding. The HFC diet increased inflammatory cells at 2 weeks, but not thereafter. This diet appeared to increase ballooned hepatocytes at 2 weeks, and they significantly increased at 8 and 14 weeks. Thus, NAS were already around 5.0 by the HFC diet for 2 weeks, and continued to increase with longer duration of the diet. The SP diet did not influence the fibrosis score, whereas the HFC diet significantly increased it in a duration-dependent manner.

Hepatic lipids

Because lipid accumulations were histopathologically observed in the liver of the rats fed the HFC diet, we measured hepatic TG and TC levels. The SP diet did not influence hepatic TG and TC levels throughout the observed periods (Fig. 4a-b). Hepatic TG levels in rats fed the HFC diet were significantly higher than those fed the SP diet. Long-term (14 weeks) feeding of the diet, however, decreased the TG levels compared with those in 2-week feeding. In contrast, hepatic TC levels of the rats fed the HFC diet were considerably higher than those in the SP diet, and the levels increased with the increasing duration of the feeding. 


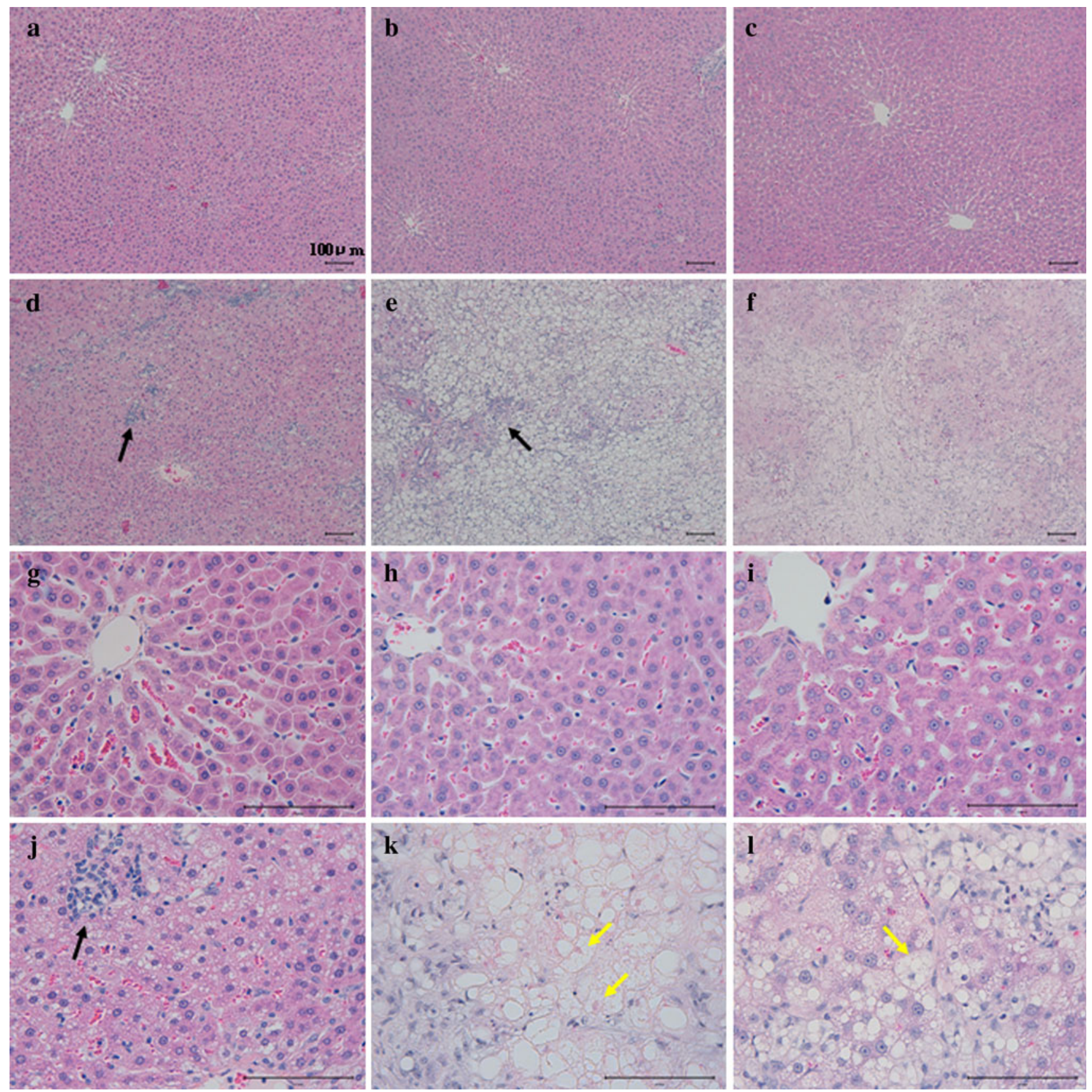

Fig. 2 H\&E staining of liver in rats fed SP and HFC diets [original magnifications were $\times 100(\mathbf{a}-\mathbf{f})$ and $\times 400(\mathbf{g}-\mathbf{l})]$. Liver from rats fed SP diet for $2(\mathbf{a}, \mathbf{g}), 8(\mathbf{b}, \mathbf{h})$ and 14 weeks $(\mathbf{c}, \mathbf{i})$; liver from rats fed HFC diet for $2(\mathbf{d}, \mathbf{j}), 8(\mathbf{e}, \mathbf{k})$, and 14 weeks $(\mathbf{f}, \mathbf{l})$. Lipid deposition (microvesicular steatosis predominantly) and focal or spotty necrosis with lymphocyte infiltrations (arrows) were observed in the liver of rats fed the HFC diet for 2 weeks (d, j). Extensive lipid deposition (macrovesicular steatosis predominantly) and multilobular necrosis in the liver of rats fed the HFC diet for 8 weeks $(\mathbf{e}, \mathbf{k})$. In addition to these findings, fibrosis was suspected in the liver of rats fed the HFC diet for 14 weeks (f, l). Mallory-Denk bodies (yellow arrows) were also observed in ballooned hepatocytes from rats fed the HFC diet for 8 and 14 weeks $(\mathbf{k}, \mathbf{l})$

\section{Discussion}

In this study, we demonstrated that such a diet, referred to as the HFC diet, caused severe lipid accumulation and mild inflammation with an NAS evaluation around 5.0 in 2 weeks in the liver of SHRSP5/Dmcr rats. In addition to these observations, we noted ballooned hepatocytes with Mallory-Denk bodies and mild fibrosis at 8 weeks of 

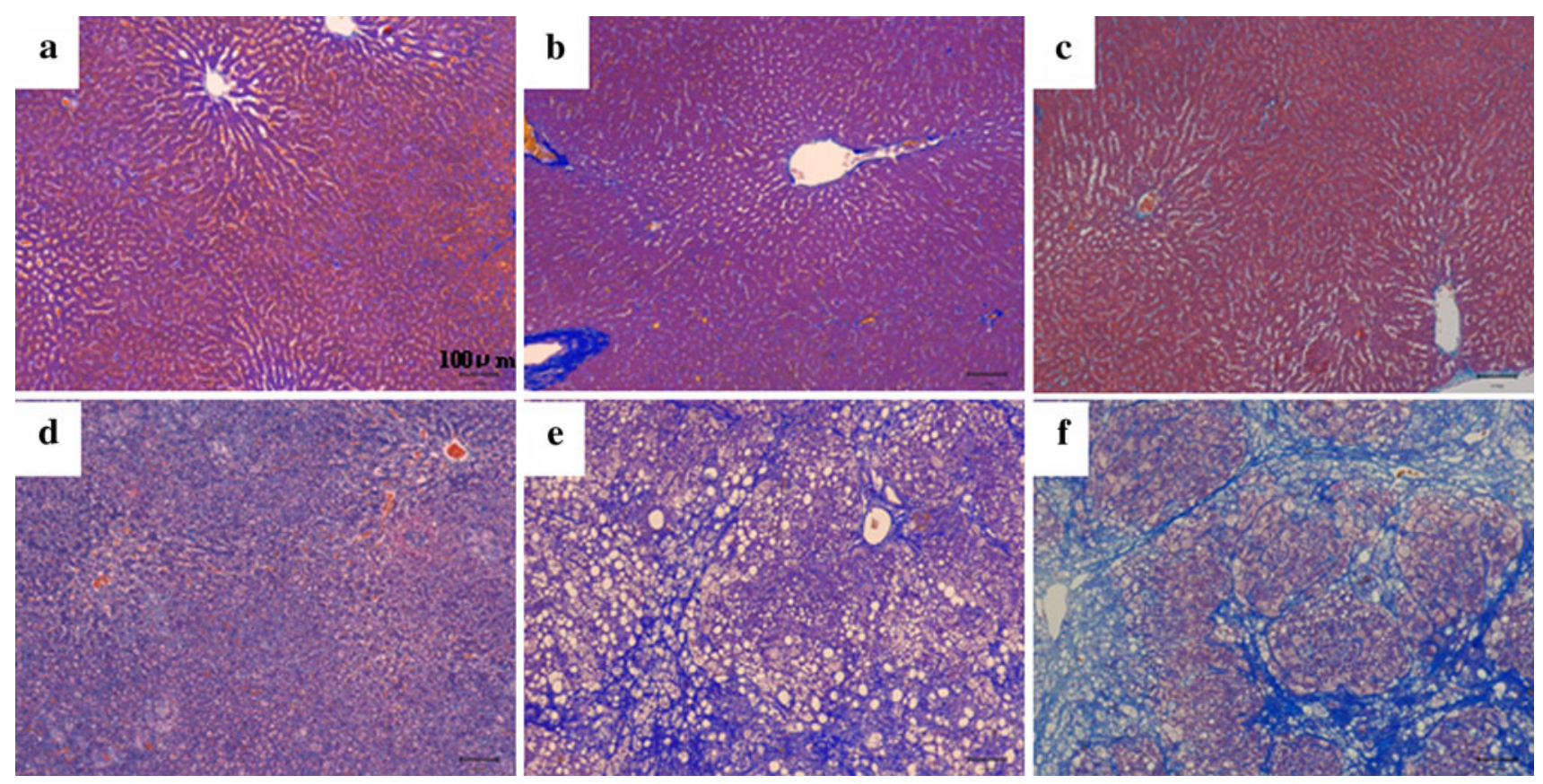

Fig. 3 Azan staining of liver sections from each group (original magnifications $\times 100$ ). The livers of rats fed the SP diet for $2(\mathbf{a}), 8$ (b), and 14 weeks (c), and HFC diet for 2 (d), 8 (e), and 14 weeks (f).
Extensive fibrosis (arrows) was evident in the liver of the rats fed the HFC diet for 8 weeks (e), and fiber septa of honeycomb fibrosis (arrows) was evident in the liver of the rats fed the diet for 14 weeks (f)

Table 3 NAS and fibrosis scores

\begin{tabular}{llllllcc}
\hline Diet & $\begin{array}{l}\text { Duration } \\
\text { (weeks) }\end{array}$ & Total NAS & Steatosis & $\begin{array}{l}\text { Lobular } \\
\text { inflammation } \\
(0-3)\end{array}$ & $\begin{array}{l}\text { Hepatocyte } \\
\text { ballooning } \\
(0-2)\end{array}$ & $\begin{array}{l}\text { Macrovesicular } \\
\text { steatosis } \\
(0-3)\end{array}$ & $\begin{array}{l}\text { Fibrosis stage } \\
(0-4)\end{array}$ \\
\hline SP & 2 & $(0-8)$ & $(0-3)$ & $0 \pm 0.00$ & $0 \pm 0.00$ & $0 \pm 0.00$ \\
SP & 8 & $0.8 \pm 0.10$ & $0.2 \pm 0.16$ & $0.6 \pm 0.14$ & $0 \pm 0.00$ & $0 \pm 0.00$ & $0 \pm 0.00$ \\
SP & 14 & $1.0 \pm 0.41$ & $0.1 \pm 0.12$ & $0.9 \pm 0.41$ & $0 \pm 0.08$ & $0 \pm 0.00$ & $0 \pm 0.00$ \\
HFC & 2 & $4.9 \pm 1.10^{\mathrm{a}}$ & $2.9 \pm 0.14^{\mathrm{a}}$ & $1.3 \pm 0.53$ & $0.7 \pm 1.17$ & 0.00 & $0.3 \pm 0.32^{\mathrm{a}}$ \\
$\mathrm{HFC}$ & 8 & $5.1 \pm 0.85^{\mathrm{a}}$ & $3.0 \pm 0.00^{\mathrm{a}}$ & $1.3 \pm 0.43^{\mathrm{a}}$ & $0.9 \pm 0.45^{\mathrm{a}}$ & $1.6 \pm 0.64^{\mathrm{a}, \mathrm{b}}$ & $2.5 \pm 0.79^{\mathrm{a}, \mathrm{b}}$ \\
HFC & 14 & $5.7 \pm 0.86^{\mathrm{a}}$ & $3.0 \pm 0.00^{\mathrm{a}}$ & $1.9 \pm 0.47^{\mathrm{a}}$ & $0.7 \pm 0.51^{\mathrm{a}}$ & $2.0 \pm 0.81^{\mathrm{a}, \mathrm{b}}$ & $3.0 \pm 0.07^{\mathrm{a}, \mathrm{b}}$ \\
\hline
\end{tabular}

Total NAS indicates the sum of steatosis, lobular inflammation, and hepatocyte ballooning scores. Each figure represents the mean $\pm \operatorname{SD}(n=6)$

a Significant differences were observed between the respective SP and HFC diets $(p<0.05)$

b Significantly different from the respective rats fed each diet for 2 weeks $(p<0.05)$

feeding, and more macrovesicular steatosis and honeycomb fibrosis formations of different sizes along with bridging necrosis at 14 weeks. These histopathological findings were confirmed to be very similar to those observed during the progression of NASH in patients $[10,11]$. The ballooned hepatocytes and Mallory-Denk bodies were thought to be markers for NASH [10, 24]. According to the fibrosis classification [21], hepatic histopathological changes were designated as stage 2 in the rats fed the HFC diet for 8 weeks, and stage 3 in those on the diet for 14 weeks. Thus, HFC diet really induced severe fibrosis in SHRSP5/ Dmcr rats, which could hardly be induced in the other rodents [8]. It is of considerable interest that patients with nonobese and nondiabetic NASH generally consumed high amounts of cholesterol and saturated fatty acid [25].

Insulin resistance and obesity are common features in patients with NAFLD [26]. Collison et al. [27] reported that the SHRSP rat itself had insulin resistance, importantly suggesting that SHRSP5/Dmcr rats may also have this resistance. However, SHRSP5/Dmcr rats did not exhibit obesity, and the increase in their body weight was rather small. Carbohydrate intake was quite small in the HFC diet compared to the SP diet. Indeed, serum glucose levels were significantly lower in the HFC-diet group than those in the 

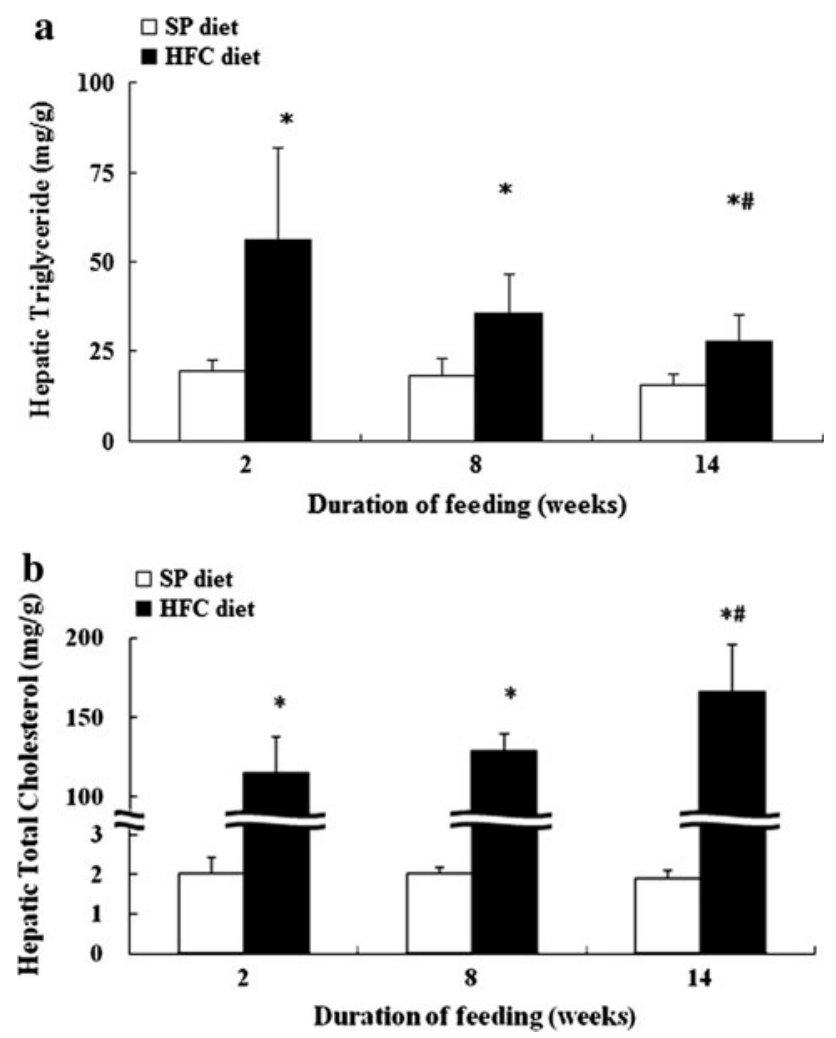

Fig. 4 TG (a) and TC (b) levels in the liver. Open square shows rats fed the SP diet, and closed square shows rats fed the HFC diet. Values are expressed as the mean $\pm \mathrm{SD}(n=6)$. *Significant differences were observed between rats fed SP and HFC diets $(p<0.05)$

SP diet group. Thus, the HFC diet group did not increase the HOMA-IR values. Additionally, blood pressure in HFC diet groups was not higher than that of the SP diet groups, which may be, in part, because of the decreased sodium intake in the former. Nevertheless, the diet did induce ballooned hepatocytes with Mallory-Denk bodies and severe fibrosis in the liver of SHRSP5/Dmcr rats, suggesting that the HFC diet may lead to hepatitis and severe fibrosis in some cases independently of insulin resistance. We could not clarify the effect of latent insulin resistance in SHRSP5/Dmcr.

Matteoni et al. [2] classified NAFLD into four categories: type 1 (fatty liver alone), type 2 (fat accumulation and lobular inflammation), type 3 (fat accumulation and ballooning degeneration) and type 4 (fat accumulation, ballooning degeneration, and either Mallory hyaline or fibrosis). According to this classification, the liver of rats fed the HFC diet for 2 weeks was already classified as type 2 , then as type 3 or type 4 by 8 weeks, while that by 14 weeks was obviously type 4 . These results again suggest that in SHRSP5/Dmcr rats, the HFC diet induced all pathological stages classified by Matteoni et al. [2] during 14 weeks.
There are many reports concerning animal models of steatohepatitis or fibrosis. In these cases, several kinds of high-fat diet or methionine- and choline-deficient diet have been used [8,9], and lipid infiltration, inflammation, and activation of stellate cells were found to occur during the steatohepatitis progression. Thus, these animal models were shown to be of benefit to clarify the mechanism, but could not develop typical fibrosis such as honeycomb network in different nodular sizes.

On the other hand, animal models of DMN or $\mathrm{CCl}_{4}$ administration have been used for analyzing the mechanism of hepatic fibrosis seen in patients with NASH [12-14]. In these animal models, the portion of typical fibrosis with honeycomb network formed with different sizes of nodule was located at the area of the stellate cells, suggesting a relationship between stellate cell activation and fibrosis. Although the mechanism of fibrosis has been partially solved using these chemically induced animal models, the relation to the progression of steatosis and inflammation, which are usually observed in patients with $\mathrm{NASH}$, remains unclear. Recently, $\mathrm{CCl}_{4}$ was administered to nuclear factor-kappa B1 (p50) knockout mice twice weekly for 12 weeks to investigate the mechanism of inflammation and fibrosis [28]. In this animal model, both inflammation and fibrosis were induced, but the characteristic honeycomb fibrosis could not be induced. The influence of nutritional factors was not considered in these animal models.

More recently, another animal model to evaluate the mechanism of NASH progression has been developed. When phosphatase and tensin homolog-deficient mice were killed at 40 weeks of age [29, 30], the steatohepatitis, Mallory-Denk bodies, and sinusoidal fibrosis observed were very similar to the histopathological findings of human NASH [10, 11, 24]. However, in this mouse model, the characteristic honeycomb fibrosis was not observed at that age.

In contrast, our established animal model was characterized by developing steatosis and inflammation, hepatocyte ballooning with Mallory-Denk bodies, and honeycomb fibrosis at only 14 weeks of HFC feeding, suggesting the SHRSP5/Dmcr rat could be a unique experimental model exhibiting the overall pathology of steatohepatitis and fibrosis progression just by simple dietary manipulation.

The feeding of the HFC diet decreased the serum albumin levels in the rats at 8 and 14 weeks. In humans, the decrease is also reported with increasing serum type IV collagen $7 \mathrm{~S}$ levels [5]. In the rats fed the HFC diet, the decrease in albumin level was preceded by an increase in type IV collagen $7 \mathrm{~S}$ at 14 weeks. However, it should be noted that the consumption of protein in rats fed the HFC diet might be lower than that on the SP diet. Although serum AST, ALT, and TC levels were increased at 2 weeks 
on the HFC diet, serum type IV collagen $7 \mathrm{~S}$ increased only at 14 weeks, somewhat behind the histopathological findings. Taken together, serum AST and ALT and/or TC levels may be good biomarkers for the steatohepatitis progression, because in patients diagnosed as NASH, these elements have been reported to increase [31], though TC levels may not be always increased in the patients [25].

In our results, the increase in hepatic TG levels after feeding of HFC diet for 2 weeks decreased along with the continual feeding of the diet for 14 weeks. Yamaguchi et al. [32] reported that diacylglycerol acyltransferase 2 inhibitor, which inhibits the synthesis of TG from fatty acids, exacerbated oxidative stress and fibrosis. Therefore, they suggested that TG itself may not be hepatotoxic, and may have a role in preventing progressive liver damage. In our steatohepatitis and severe fibrosis novel model rats, the decrease in the hepatic TG with HFC diet may be related to the progression of fibrosis. Of course, further study is warranted. Serum TG levels also did not increase at 2 and 8 weeks in HFC-diet group regardless of the great amount of diet intake. The total hepatic TG levels of SHRSP5/ Dmcr rats fed the HFC diet exponentially increased $(142 \pm 33.4,152 \pm 59.9$, and $136 \pm 26.6 \mathrm{mg} /$ liver in SP diet for 2,8 , and 14 weeks, respectively; $632 \pm 287$, $1,243 \pm 454$, and $1,051 \pm 373 \mathrm{mg} /$ liver in HFC diet for 2 , 8 , and 14 weeks, respectively) following the extreme increase in liver weights. This would suggest that serum FFA might be used for the synthesis of hepatic TG. Since microsomal triglyceride transporter protein, the transporter protein of TG from liver to blood, was not induced by the HFC diet, TG in the serum could not be increased by the feeding. Thus, serum FFA might be used to synthesize of TG at 8 weeks in the liver of the HFC-diet group, which may be, in part, owing to the decrease in the serum level. Of course, further elucidation of the molecular mechanism is warranted in a future study.

In contrast, not only serum but also the hepatic TC level increased with the progression of steatohepatitis and fibrosis in the rats fed the HFC diet. After feeding for 14 weeks, the serum level was more than $1,500 \mathrm{mg} / \mathrm{dl}$. Serum TC levels are generally high in patients with NAFLD [33] or NASH [34]. The synthesis of sterol regulatory element-binding protein-2- and steroidogenic acute regulatory protein-mRNA were higher in NASH than in nonNASH individuals [35], suggesting an enhancement of TC synthesis in the patients. On the other hand, there is a report that the serum TC value cannot be related to the severity of fibrosis in human NASH [36]. Thus, the role of TC should be further investigated in the progress of NASH with advanced fibrosis. In SHRSP5/Dmcr rats used in this experiment, high intake of cholesterol may influence not only TC synthesis but also the metabolism and excretion in the liver (Naito et al., in preparation).
In our study, HFC diet time-dependently increased serum $\mathrm{TNF} \alpha$, which is important in the procession of inflammation and fibrosis: HFC diet may down-regulate $5^{\prime}$ AMP-activated protein kinase and peroxisome proliferator-activated receptor $\alpha$ signaling via TNF $\alpha$ [37], and thereby induce inflammation (nuclear factor-kappa B) and fibrosis (transforming growth factor $\beta$, smooth muscle actin $\alpha$, etc.). Although serum adiponectin levels increased 2 weeks after feeding of the HFC diet, the levels decreased thereafter. In contrast, serum adiponectin levels were reported to decrease with feeding of the high-fat diet throughout the observed periods (for 1, 2, 4, and 8 weeks) [38]. We could not clarify why adiponectin levels increased after feeding of the HFC diet for 2 weeks in this experiment, and further study including the molecular mechanism is needed. The key point is that the increased serum adiponectin levels were reproducibly observed. Additionally, the levels appeared not to be associated with liver TG in C57B1/6 mice [39].

In conclusion, we have developed a unique animal model of steatohepatitis and fibrosis progression as observed in patients with NASH using SHRSP5/Dmcr rats fed an HFC diet. This model may be useful to investigate the mechanism and treatment of human NASH without obesity.

Acknowledgments This work was supported by a Grant-in-Aid for Young Scientists B (20700603) and Scientific Research (23390161) from the Japan Society for the Promotion of Science.

\section{References}

1. Marchesini G, Brizi M, Bianchi G, Tomassetti S, Bugianesi E, Lenzi M, et al. Nonalcoholic fatty liver disease: a feature of the metabolic syndrome. Diabetes. 2001;50:1844-50.

2. Matteoni CA, Younossi ZM, Gramlich T, Boparai N, Liu YC, McCullough AJ. Nonalcoholic fatty liver disease: a spectrum of clinical and pathological severity. Gastroenterology. 1999;116: 1413-9.

3. Shimada M, Hashimoto E, Taniai M, Hasegawa K, Okuda H, Hayashi N, et al. Hepatocellular carcinoma in patients with nonalcoholic steatohepatitis. J Hepatol. 2002;37:154-60.

4. Hashimoto E, Yatsuji S, Tobari M, Taniai M, Torii N, Tokushige $\mathrm{K}$, et al. Hepatocellular carcinoma in patients with nonalcoholic steatohepatitis. J Gastroenterol. 2009;44:89-95.

5. Sakugawa H, Nakayoshi T, Kobashigawa K, Yamashiro T, Maeshiro T, Miyagi S, et al. Clinical usefulness of biochemical markers of liver fibrosis in patients with nonalcoholic fatty liver disease. World J Gastroenterol. 2005;11:255-9.

6. Hashimoto E, Yatsuji S, Kaneda H, Yoshioka Y, Taniai M, Tokushige $\mathrm{K}$, et al. The characteristics and natural history of Japanese patients with nonalcoholic fatty liver disease. Hepatol Res. 2005;33:72-6.

7. Matsuzawa N, Takamura T, Kurita S, Misu H, Ota T, Ando H, et al. Lipid-induced oxidative stress causes steatohepatitis in mice fed an atherogenic diet. Hepatology. 2007;46:1392-403.

8. Cong WN, Tao RY, Tian JY, Liu GT, Ye F. The establishment of a novel non-alcoholic steatohepatitis model accompanied with obesity and insulin resistance in mice. Life Sci. 2008;82:983-90. 
9. Ip E, Farrell G, Hall P, Robertson G, Leclercq I. Administration of the potent PPARalpha agonist, Wy-14, 643, reverses nutritional fibrosis and steatohepatitis in mice. Hepatology. 2004;39: 1286-96.

10. Yeh MM, Brunt EM. Pathology of nonalcoholic fatty liver disease. Am J Clin Pathol. 2007;128:837-47.

11. Tseng PH, Liu CJ, Kao JH, Shun CT, Chen PJ, Chen DS. Disease progression in a patient with nonalcoholic steatohepatitis. J Formos Med Assoc. 2008;107:816-21.

12. Li CH, Piao DM, Xu WX, Yin ZR, Jin JS, Shen ZS. Morphological and serum hyaluronic acid, laminin and type IV collagen changes in dimethylnitrosamine-induced hepatic fibrosis of rats. World J Gastroenterol. 2005;11:7620-4.

13. Son G, Iimuro Y, Seki E, Hirano T, Kaneda Y, Fujimoto J. Selective inactivation of NF-kappaB in the liver using NFkappaB decoy suppresses CCl4-induced liver injury and fibrosis. Am J Physiol Gastrointest Liver Physiol. 2007;293:G631-9.

14. Wang XH, Zhao J, Zhang WG, Zhang LY, Ma RQ, Wang LQ, et al. Scanning electron microscopic observation: three-dimensional architecture of the collagen in hepatic fibrosis rats. Chin Med J (Engl). 2007;120:308-12.

15. Yamori Y. Selection of arteriolipidosis-prone rats (ALR). Jpn Heart J. 1977;18:602-3.

16. Yamori Y, Kitamura Y, Nara Y, Iritani N. Mechanism of hypercholesterolemia in arteriolipidosis-prone rats (ALR). Jpn Circ J. 1981;45:1068-73.

17. Sansawa H, Takahashi M, Tsuchikura S, Endo H. Effect of chlorella and its fractions on blood pressure, cerebral stroke lesions, and life-span in stroke-prone spontaneously hypertensive rats. J Nutr Sci Vitaminol. 2006;52:457-66.

18. Folch J, Lees M, Sloane Stanley GH. A simple method for the isolation and purification of total lipides from animal tissues. J Biol Chem. 1957;226:497-509.

19. Kleiner DE, Brunt EM, Van Natta M, Behling C, Contos MJ, Cummings OW, et al. Design and validation of a histological scoring system for nonalcoholic fatty liver disease. Hepatology. 2005;41:1313-21.

20. Elias J Jr, Altun E, Zacks S, Armao DM, Woosley JT, Semelka RC. MRI findings in nonalcoholic steatohepatitis: correlation with histopathology and clinical staging. Magn Reson Imaging. 2009;27:976-87.

21. Brunt EM, Janney CG, Di Bisceglie AM, Neuschwander-Tetri BA, Bacon BR. Nonalcoholic steatohepatitis: a proposal for grading and staging the histological lesions. Am J Gastroenterol. 1999;94:2467-74.

22. Gressner OA, Weiskirchen R, Gressner AM. Biomarkers of liver fibrosis: clinical translation of molecular pathogenesis or based on liver-dependent malfunction tests. Clin Chim Acta. 2007;381: 107-13.

23. Fisher CD, Lickteig AJ, Augustine LM, Oude Elferink RP, Besselsen DG, Erickson RP, et al. Experimental non-alcoholic fatty liver disease results in decreased hepatic uptake transporter expression and function in rats. Eur J Pharmacol. 2009;613: 119-27.

24. Zatloukal K, French SW, Stumptner C, Strnad P, Harada M, Toivola DM, Cadrin M, Omary MB. From Mallory to Mallory-Denk bodies: what, how and why? Exp Cell Res. 2007;313(10):2033-49.

25. Musso G, Gambino R, De Michieli F, Cassader M, Rizzetto M, Durazzo $\mathrm{M}$, et al. Dietary habits and their relations to insulin resistance and postprandial lipemia in nonalcoholic steatohepatitis. Hepatology. 2003;37:909-16.

26. Brunt EM, Kleiner DE, Wilson LA, Unalp A, Behling CE, Lavine JE, et al. Portal chronic inflammation in nonalcoholic fatty liver disease (NAFLD): a histologic marker of advanced NAFLDClinicopathologic correlations from the nonalcoholic steatohepatitis clinical research network. Hepatology. 2009;49:809-20.

27. Collison M, Glazier AM, Graham D, Morton JJ, Dominiczak $\mathrm{MH}$, Aitman TJ, et al. Cd36 and molecular mechanisms of insulin resistance in the stroke-prone spontaneously hypertensive rat. Diabetes. 2000;49:2222-6.

28. Oakley F, Mann J, Nailard S, Smart DE, Mungalsingh N, Constandinou C, et al. Nuclear factor-kappaB1 (p50) limits the inflammatory and fibrogenic responses to chronic injury. Am J Pathol. 2005;166:695-708.

29. Watanabe S, Horie Y, Kataoka E, Sato W, Dohmen T, Ohshima $\mathrm{S}$, et al. Non-alcoholic steatohepatitis and hepatocellular carcinoma: lessons from hepatocyte-specific phosphatase and tensin homolog (PTEN)-deficient mice. J Gastroenterol Hepatol. 2007;22:S96-100.

30. Ishii H, Horie Y, Ohshima S, Anezaki Y, Kinoshita N, Dohmen $\mathrm{T}$, et al. Eicosapentaenoic acid ameliorates steatohepatitis and hepatocellular carcinoma in hepatocyte-specific Pten-deficient mice. J Hepatol. 2009;50:562-71.

31. Sazci A, Akpinar G, Aygun C, Ergul E, Senturk O, Hulagu S. Association of apolipoprotein E polymorphisms in patients with non-alcoholic steatohepatitis. Dig Dis Sci. 2008;53:3218-24.

32. Yamaguchi K, Yang L, McCall S, Huang J, Yu XX, Pandey SK, et al. Inhibiting triglyceride synthesis improves hepatic steatosis but exacerbates liver damage and fibrosis in obese mice with nonalcoholic steatohepatitis. Hepatology. 2007;45:1366-74.

33. Fu CC, Chen MC, Li YM, Liu TT, Wang LY. The risk factors for ultrasound-diagnosed non-alcoholic fatty liver disease among adolescents. Ann Acad Med Singap. 2009;38:15-21.

34. Puri P, Baillie RA, Wiest MM, Mirshahi F, Choudhury J, Cheung $\mathrm{O}$, et al. A lipidomic analysis of nonalcoholic fatty liver disease. Hepatology. 2007;46:1081-90.

35. Caballero F, Fernández A, De Lacy AM, Fernández-Checa JC, Caballería J, García-Ruiz C. Enhanced free cholesterol, SREBP-2 and StAR expression in human NASH. J Hepatol. 2009;50: 789-96.

36. Angulo $\mathrm{P}$, Keach JC, Batts KP, Lindor KD. Independent predictors of liver fibrosis in patients with nonalcoholic steatohepatitis. Hepatology. 1999;30:1356-62.

37. Moriya T, Naito H, Ito Y, Nakajima T. "Hypothesis of seven balances": molecular mechanisms behind alcoholic liver diseases and association with PPARalpha. J Occup Health. 2009;51(5): 391-403.

38. Yun JW, Shin ES, Cho SY, Kim SH, Kim CW, Lee TR, et al. The effects of BADGE and caffeine on the time-course response of adiponectin and lipid oxidative enzymes in high fat diet-fed C57BL/6 J mice: correlation with reduced adiposity and steatosis. Exp Anim. 2008;57(5):461-9.

39. Duval C, Thissen U, Keshtkar S, Accart B, Stienstra R, Boekschoten MV, et al. Adipose tissue dysfunction signals progression of hepatic steatosis towards nonalcoholic steatohepatitis in C57BL/6 mice. Diabetes. 2010;59(12):3181-91. 